\title{
POSITIVE ENERGY REPRESENTATIONS FOR LOCALLY FINITE SPLIT LIE ALGEBRAS
}

\author{
TIMOTHÉE MARQUIS* AND KARL-HERMANN NEEB ${ }^{\dagger}$
}

\begin{abstract}
Let $\mathfrak{g}$ be a locally finite split simple complex Lie algebra of type $A_{J}, B_{J}, C_{J}$ or $D_{J}$ and $\mathfrak{h} \subseteq \mathfrak{g}$ be a splitting Cartan subalgebra. Fix $D \in \operatorname{der}(\mathfrak{g})$ with $\mathfrak{h} \subseteq \operatorname{ker} D$ (a diagonal derivation). Then every unitary highest weight representation $\left(\rho_{\lambda}, V^{\lambda}\right)$ of $\mathfrak{g}$ extends to a representation $\tilde{\rho}_{\lambda}$ of the semidirect product $\mathfrak{g} \rtimes \mathbb{C} D$ and we say that $\tilde{\rho}_{\lambda}$ is a positive energy representation if the spectrum of $-i \tilde{\rho}_{\lambda}(D)$ is bounded from below. In the present note we characterise all pairs $(\lambda, D)$ with $\lambda$ bounded for which this is the case.

If $U_{1}(\mathcal{H})$ is the unitary group of Schatten class 1 on an infinite dimensional real, complex or quaternionic Hilbert space and $\lambda$ is bounded, then we accordingly obtain a characterisation of those highest weight representations $\pi_{\lambda}$ satisfying the positive energy condition with respect to the continuous $\mathbb{R}$-action induced by $D$. In this context the representation $\pi_{\lambda}$ is norm continuous and our results imply the remarkable result that, for positive energy representations, adding a suitable inner derivation to $D$, we can achieve that the minimal eigenvalue of $\tilde{\rho}_{\lambda}(D)$ is 0 (minimal energy condition). The corresponding pairs $(\lambda, D)$ satisfying the minimal energy condition are rather easy to describe explicitly.
\end{abstract}

\section{INTRODUCTION}

Locally finite split Lie algebras are natural infinite-dimensional generalisations of finite-dimensional Lie algebras. More precisely, a Lie algebra $\mathfrak{g}$ over a field $\mathbb{K}$ of characteristic zero is called split if it has a root decomposition $\mathfrak{g}=\mathfrak{h}+\sum_{\alpha \in \Delta} \mathfrak{g}_{\alpha}$ with respect to some maximal abelian subalgebra $\mathfrak{h}$. It is moreover locally finite if every finite subset of $\mathfrak{g}$ generates a finite-dimensional subalgebra. Such a Lie algebra $\mathfrak{g}$ possesses a generalised Levi decomposition (see [Stu99]), whose Levi factor is an $\mathfrak{h}$-invariant semisimple Lie algebra. In turn, the infinite-dimensional locally finite split simple Lie algebras have been classified (see [NS01), and can be realised as subalgebras of the algebra $\mathfrak{g l}(J, \mathbb{K})$ of $J \times J$ matrices with only finitely many nonzero entries, for some infinite set $J$. They fall into four distinct families of isomorphism classes, parametrised by the locally finite root systems of type $A_{J}, B_{J}, C_{J}$ and $D_{J}$ (see [LN04]).

On the other hand, a locally finite split simple Lie algebra $\mathfrak{g}$ is the directed union of its finitedimensional simple subalgebras ( $\underline{\text { Stu99, }}$, Section V]). Unitary highest weight representations for such Lie algebras over $\mathbb{K}=\mathbb{C}$ were studied in $\mathbf{N e e 9 8}$. By [Stu99, Section VIII], $\mathfrak{g}$ carries an antilinear involutive antiautomorphism $X \mapsto X^{*}$ such that $\mathfrak{g}_{\mathbb{R}}=\left\{X \in \mathfrak{g} \mid X^{*}=-X\right\}$ is a compact real form, that is, $\mathfrak{g}_{\mathbb{R}}$ is a union of finite dimensional compact Lie algebras. A $\mathfrak{g}$-module $V$ is then called unitary if it carries a contravariant positive definite hermitian form $\langle\cdot, \cdot\rangle$, in the sense that $\langle X . v, w\rangle=\left\langle v, X^{*} . w\right\rangle$ for all $v, w \in V, X \in \mathfrak{g}$. A $\mathfrak{g}$-module $V=V^{\lambda}$ is called a highest weight module with highest weight $\lambda \in \mathfrak{h}^{*}$ (with respect to some positive system $\Delta_{+} \subseteq \Delta$ of roots) if it is generated by some primitive element, that is, by some $\mathfrak{h}$-weight vector $v \in V$ with weight $\lambda$ such that $\mathfrak{g}_{\alpha} \cdot v=\{0\}$ for all $\alpha \in \Delta_{+}$. Unitary highest weight modules $V^{\lambda}$ for $\mathfrak{g}$ were classified in [Nee98, and correspond to dominant integral weights $\lambda$. Moreover, if $W \leq \mathrm{GL}\left(\mathfrak{h}^{*}\right)$ denotes the Weyl group of $\mathfrak{g}$, the set $\mathcal{P}_{\lambda}$ of $\mathfrak{h}$-weights on $V^{\lambda}$ is given by $\mathcal{P}_{\lambda}=\operatorname{conv}(W \cdot \lambda) \cap(\lambda+\mathbb{Z}[\Delta])([$ Nee98, Theorem I.11] $)$.

In this paper, we characterise the unitary highest weight representations of $\mathfrak{g}$ satisfying the following "positive energy condition". A skew-hermitian derivation $D \in \operatorname{der}(\mathfrak{g})$ of $\mathfrak{g}$ is called graded if it annihilates $\mathfrak{h}$, in which case $D$ preserves the root spaces of $\mathfrak{g}$. Such a derivation is then described by a

* Supported by a Marie Curie Intra-European Fellowship.

†Supported by DFG-grant NE 413/7-2, Schwerpunktprogramm "Darstellungstheorie". 
character $\chi: \mathbb{Z}[\Delta] \rightarrow \mathbb{R}$ such that $D\left(x_{\alpha}\right)=i \chi(\alpha) x_{\alpha}$ for all $x_{\alpha} \in \mathfrak{g}_{\alpha}, \alpha \in \Delta$. Any unitary highest weight representation $\rho_{\lambda}: \mathfrak{g} \rightarrow \operatorname{End}\left(V^{\lambda}\right)$ of $\mathfrak{g}$ may be extended to a representation $\widetilde{\rho}_{\lambda}: \mathfrak{g} \rtimes \mathbb{C} D \rightarrow \operatorname{End}\left(V^{\lambda}\right)$ by setting $\widetilde{\rho}_{\lambda}(D) v_{\mu}=i \chi(\mu-\lambda) v_{\mu}$ for any $\mu \in \mathcal{P}_{\lambda}$ and any weight vector $v_{\mu} \in V^{\lambda}$ of weight $\mu$. The representation $\widetilde{\rho}_{\lambda}$ is called a positive energy representation if the spectrum of $H:=-i \widetilde{\rho}_{\lambda}(D)$ is bounded from below. We call $H$ a Hamiltonian of the representation $\rho_{\lambda}$.

In view of the above description of $\mathcal{P}_{\lambda}$, the positive energy condition can then be rewritten as

$$
\inf \chi(W \cdot \lambda-\lambda)>-\infty \text {. }
$$

Let $J$ be an infinite set such that $\Delta$ is of one of the types $A_{J}, B_{J}, C_{J}$ or $D_{J}$. Then there exists some basis $\left(e_{j}\right)_{j \in J}$ of $\mathfrak{h}$ (respectively a one-dimensional extension of $\mathfrak{h}$ ) indexed by $J$ such that the set of coroots $\Delta^{\vee}=\left\{\alpha^{\vee} \mid \alpha \in \Delta\right\}$ (see [Stu99, Section I]) is contained in $\operatorname{span}_{\mathbb{Z}}\left(e_{j}\right)_{j \in J}$, while $\Delta$ is contained in the $\mathbb{Z}$-span of the linearly independent system $\left(\epsilon_{j}\right)_{j \in J} \subseteq \mathfrak{h}^{*}$ defined by $\epsilon_{j}\left(e_{k}\right)=\delta_{j k}$ for all $j, k \in J$ (see [NS01, Section I and Theorem IV.6]).

We recall that the weight $\lambda \in \mathfrak{h}^{*}$ is called integral if $\lambda\left(\alpha^{\vee}\right) \in \mathbb{Z}$ for all $\alpha \in \Delta$. This implies in particular that $\lambda$ is discrete, in the sense that $\left\{\lambda_{j}:=\lambda\left(e_{j}\right) \mid j \in J\right\}$ is a discrete subset of $\mathbb{R}$. We moreover call $\lambda$ bounded if $\sup _{j \in J}\left|\lambda_{j}\right|<\infty$. Finally, a character $\chi: \mathbb{Z}[\Delta] \rightarrow \mathbb{R}$ is said to be summable if it is the restriction of a homomorphism $\tilde{\chi}: \operatorname{span}_{\mathbb{Z}}\left(\epsilon_{j}\right)_{j \in J} \rightarrow \mathbb{R}$ satisfying

$$
\sum_{j \in J}\left|\widetilde{\chi}\left(\epsilon_{j}\right)\right|<\infty
$$

We can now state the main result of this paper, which provides a characterisation of the positive energy highest weight representations of $\mathfrak{g}$ with bounded highest weight.

Theorem A. Let $(\mathfrak{g}, \mathfrak{h})$ be a locally finite split simple Lie algebra with root system $\Delta$ and Weyl group $W \leq \mathrm{GL}\left(\mathfrak{h}^{*}\right)$. Let $\lambda \in \mathfrak{h}^{*}$ be discrete and bounded. Then for a character $\chi: \mathbb{Z}[\Delta] \rightarrow \mathbb{R}$, the following are equivalent:

(1) $\inf \chi(W . \lambda-\lambda)>-\infty$.

(2) $\chi=\chi_{\min }+\chi_{\text {sum }}$ for some "minimal energy" character $\chi_{\min }$, satisfying $\inf \chi_{\min }(W \cdot \lambda-\lambda)=0$, and some summable character $\chi_{\text {sum. }}$.

We also provide a description of "minimal energy" characters (see Proposition 5.3 and Remark 5.9). In [HN12, Corollary 3.2] a similar description of the "minimal energy" characters was obtained by Coxeter geometry. A priori, these can be described much more easily than the "positive energy" characters and the main point of the theorem is that it reduces the latter problem to the former. The proof of Theorem $\mathrm{A}$ is given in Section 6 below.

The assumption that the highest weight $\lambda$ be bounded in Theorem $\mathrm{A}$ is motivated by the study of positive energy (projective) unitary representations of the corresponding Lie groups, which we now briefly review. We then state a corollary of Theorem $\mathrm{A}$ in this context.

The real form $\mathfrak{g}_{\mathbb{R}}$ of the locally finite split simple Lie algebra $\mathfrak{g}$ is endowed with an invariant scalar product $\langle\cdot, \cdot\rangle$, in the sense that $\langle[X, Y], Z\rangle=\langle X,[Y, Z]\rangle$ for all $X, Y, Z \in \mathfrak{g}_{\mathbb{R}}$. For instance, if $\mathfrak{g}=\mathfrak{s l}(J, \mathbb{C})$ is the subalgebra of $\mathfrak{g l}(J, \mathbb{C})$ of traceless matrices, it is given by $\langle X, Y\rangle=\operatorname{tr}\left(X Y^{*}\right)$. The Hilbert space completion $\mathfrak{k}$ of $\mathfrak{g}_{\mathbb{R}}$ is then a so-called Hilbert-Lie algebra, that is, a real Lie algebra and a real Hilbert space with compatibility of the two structures given by the invariance of the scalar product. For $\mathfrak{g}=\mathfrak{s l}(J, \mathbb{C})$, the corresponding completion is the space $\mathfrak{u}_{2}(\mathcal{H})$ of skew-symmetric Hilbert-Schmidt operators on the complex Hilbert space $\mathcal{H}=\ell^{2}(J, \mathbb{C})$.

By a theorem of Schue (Sch61]), Hilbert-Lie algebras decompose into an orthogonal direct sum of simple ideals (and center). Moreover, each simple infinite-dimensional Hilbert-Lie algebra is isomorphic to $\mathfrak{u}_{2}(\mathcal{H})$, for some infinite-dimensional real, complex or quaternionic Hilbert space $\mathcal{H}$. These are, in turn, classified by the locally finite root systems (see [Nee12, Examples C.4,5,6]).

Going back to our example $\mathfrak{g}=\mathfrak{s l}(J, \mathbb{C})$, we let $\rho_{\lambda}: \mathfrak{g} \rightarrow \operatorname{End}\left(V^{\lambda}\right)$ be, as before, a unitary highest weight representation of $\mathfrak{g}$ with highest weight $\lambda$. Then, under the assumption that $\lambda$ is bounded, the restriction of $\rho_{\lambda}$ to $\mathfrak{g}_{\mathbb{R}}$ extends to a continuous unitary representation $\rho_{\lambda}: \mathfrak{u}_{1}(\mathcal{H}) \rightarrow \mathfrak{u}\left(\mathcal{H}_{\lambda}\right)$ with $\left\|\rho_{\lambda}\right\| \leq \sup _{j \in J}\left|\lambda_{j}\right|$, where $\mathfrak{u}_{1}(\mathcal{H}) \subseteq \mathfrak{u}_{2}(\mathcal{H})$ denotes the Banach space of skew-hermitian trace-class 
operators on $\mathcal{H}$ and $\mathcal{H}_{\lambda}$ is the Hilbert space completion of $V^{\lambda}$ (see [Nee98, Proposition III.7]). Moreover, if $\lambda_{j} \in \mathbb{Z}$ for all $j \in J$, then $\rho_{\lambda}$ exponentiates to a holomorphic representation

$$
\widehat{\rho}_{\lambda}: U_{1}(\mathcal{H}) \rightarrow U\left(\mathcal{H}_{\lambda}\right)
$$

from $U_{1}(\mathcal{H})=\mathrm{GL}(\mathcal{H}) \cap\left(\mathbf{1}+\mathfrak{u}_{1}(\mathcal{H})\right)$ to the unitary group $U\left(\mathcal{H}_{\lambda}\right)$.

Assume now that the Lie group $G=U_{1}(\mathcal{H})$ is endowed with a continuous $\mathbb{R}$-action, given by a homomorphism $\alpha: \mathbb{R} \rightarrow \operatorname{Aut}(G): t \mapsto \alpha_{t}$. If $U: \mathbb{R} \rightarrow U\left(\mathcal{H}_{\lambda}\right): t \mapsto U_{t}$ is a unitary representation of $\mathbb{R}$ on $\mathcal{H}_{\lambda}$, then the map

$$
\pi_{\lambda}: G \rtimes_{\alpha} \mathbb{R} \rightarrow U\left(\mathcal{H}_{\lambda}\right):(g, t) \mapsto \rho_{\lambda}(g) U_{t}
$$

is called a covariant unitary representation of $(G, \mathbb{R}, \alpha)$ if it defines a unitary representation on $\mathcal{H}_{\lambda}$ of the semi-direct product $G \rtimes_{\alpha} \mathbb{R}$. This representation is said to be of positive energy if the spectrum of the corresponding Hamiltonian $H:=-\left.i \frac{d}{d t}\right|_{t=0} U_{t}$ is bounded below.

In our setting, one can show that $\alpha$ must be of the form

$$
\alpha_{t}(g)=e^{i t A} g e^{-i t A}
$$

for some self-adjoint bounded operator $A \in B(\mathcal{H})$. A sufficient condition for $\rho_{\lambda}$ to extend to a covariant representation of $(G, \mathbb{R}, \alpha)$ is the diagonalisability of $A$ : in this case, choosing the orthonormal basis $\left(e_{j}\right)_{j \in J}$ of $\mathcal{H}$ so that $A e_{j}=d_{j} e_{j}$ for all $j \in J$, for some $d_{j} \in \mathbb{R}$, one gets a covariant representation $\pi_{\lambda}$ as above by setting

$$
U_{t} v_{\lambda}=v_{\lambda} \quad \text { and } \quad U_{t} v_{\mu}=e^{i t \chi(\mu-\lambda)} v_{\mu}
$$

for any $t \in \mathbb{R}, \mu \in \mathcal{P}_{\lambda}$ and any $\mu$-weight vector $v_{\mu} \in \mathcal{H}_{\lambda}$, where $\chi: \mathbb{Z}[\Delta] \rightarrow \mathbb{R}$ is the character induced by the assignment $\epsilon_{j} \mapsto d_{j}$. Comparing this situation with Theorem $\mathrm{A}$, we see that the decomposition $\chi=\chi_{\min }+\chi_{\text {sum }}$ in this theorem corresponds to a decomposition $A=A_{\min }+A_{\text {sum }}$ of $A$ as a sum of two commuting (simultaneously diagonalisable) operators $A_{\text {min }}, A_{\text {sum }} \in B(\mathcal{H})$ such that $i A_{\text {sum }} \in \mathfrak{u}_{1}(\mathcal{H})$, and such that $A_{\min }$ yields a minimal energy representation $\pi_{\lambda}$, in the sense that the corresponding Hamiltonian $H_{\min }$ is non-negative (with eigenvalue 0 on the highest weight vector $v_{\lambda}$ ). Writing $\alpha^{\min }$ and $\alpha^{\text {sum }}$ for the $\mathbb{R}$-actions on $G$ induced by $A_{\text {min }}$ and $A_{\text {sum }}$ respectively, this implies that $\alpha_{t}$ differs only from $\alpha_{t}^{\text {min }}$ by an inner automorphism $\alpha_{t}^{\text {sum }}$ of $G$ commuting with $\alpha_{t}$ and $\alpha_{t}^{\min }(t \in \mathbb{R})$ : in this case, we will say that the corresponding covariant representations of $G \rtimes_{\alpha} \mathbb{R}$ and $G \rtimes_{\alpha^{\min }} \mathbb{R}$ are similar. Thus Theorem $\mathrm{A}$ has the following corollary:

Corollary B. Let $G=U_{1}(\mathcal{H})$ be endowed with a continuous $\mathbb{R}$-action $\alpha: \mathbb{R} \rightarrow \operatorname{Aut}(G)$, given by $\alpha_{t}(g)=e^{i t A} g e^{-i t A}$ for some self-adjoint bounded operator $A \in B(\mathcal{H})$. Assume that $A$ is diagonalisable. Then every positive energy covariant unitary representation $\pi_{\lambda}: G \rtimes_{\alpha} \mathbb{R} \rightarrow U\left(\mathcal{H}_{\lambda}\right)$ of $(G, \mathbb{R}, \alpha)$ as defined above is similar to a minimal energy representation.

Finally, one can show that the highest weight representation $\widehat{\rho}_{\lambda}: U_{1}(\mathcal{H}) \rightarrow U\left(\mathcal{H}_{\lambda}\right)$ extends to a projective unitary representation $U_{2}(\mathcal{H}) \rightarrow P U\left(\mathcal{H}_{\lambda}\right)$ of the Hilbert-Lie group

$$
U_{2}(\mathcal{H})=\mathrm{GL}(\mathcal{H}) \cap\left(\mathbf{1}+\mathfrak{u}_{2}(\mathcal{H})\right),
$$

and hence to a unitary representation on $\mathcal{H}_{\lambda}$ of a central extension $\widehat{U}_{2}(\mathcal{H})$ of $U_{2}(\mathcal{H})$, given at the Lie algebra level by the cocycle $\omega(X, Y)=i \lambda([X, Y])$ for $X, Y \in \mathfrak{u}_{2}(\mathcal{H})$, where we extend $\lambda$ in a natural way to a continuous linear functional on $\mathfrak{u}_{1}(\mathcal{H})$ which contains $[X, Y]$ (see Nee15). Since the continuous $\mathbb{R}$-action (1.1) on $U_{2}(\mathcal{H})$ lifts canonically to the central extension, the corresponding notion of positive energy for the associated projective covariant unitary representations of $U_{2}(\mathcal{H})$ is also described by Corollary B,

The situation discussed in this paper is a model case in which a rather detailed analysis of the positive energy condition can be carried out. For every triple $(G, \mathbb{R}, \alpha)$, where $G$ is a Lie group and $\alpha: \mathbb{R} \rightarrow \operatorname{Aut}(G)$ defines a continuous action, it is a challenging natural problem to determine the irreducible positive energy representations $(\pi, \mathcal{H})$ of the topological group $G^{\sharp}:=G \rtimes_{\alpha} \mathbb{R}$. As a consequence of the Borchers-Arveson Theorem (BR87, Theorem 3.2.46]), for any such representation, the restriction $\rho:=\left.\pi\right|_{G}$ is irreducible (see [Nee14, Theorem 2.5]) and the Hamiltonian of the extension to $G^{\sharp}$ is uniquely determined up to an additive constant determining the minimal energy level. Given 
$\alpha$, the set of irreducible positive energy representations of $G^{\sharp}$ can therefore be considered as a subset $\widehat{G}_{\alpha}$ of the set $\widehat{G}$ of equivalence classes of irreducible unitary representations of $G$ and one would like to determine this subset as explicitly as possible. In this paper this task is carried out for the subset $\widehat{G}_{h w}$ of "highest weight representations" of $G=U_{1}(\mathcal{H})$ in the case where $\alpha$ is given by conjugation with diagonal operators. Here Corollary B achieves in the Lie algebra context something similar as the Borchers-Arveson Theorem which also reduces the study of positive energy representations to minimal energy representations.

One can even show that the representation of the centrally extended group $\widehat{U}_{2}(\mathcal{H})$ extends to a group $\widehat{U}_{\text {res }}$ containing a copy of the centraliser $D$ of the diagonal operator $\operatorname{diag}\left(\left(\lambda_{j}\right)_{j \in J}\right)$ in $U(\mathcal{H})$ (see [Nee04, Theorem VII.18] for the $A_{J}$-case). Here $D$ is a finite product $\prod_{m \in \mathbb{Z}} U\left(\ell^{2}\left(J_{m}\right)\right)$ of full unitary groups where the factors correspond to the subsets $J_{m}:=\left\{j \in J \mid \lambda_{j}=m\right\}$. The corresponding extension $\widehat{\pi}$ to $\widehat{U}_{\text {res }}$ is a unitary Lie group representation for which one would like to understand the convex cone of all elements $X \in \mathfrak{u}_{\text {res }}$ in the Lie algebra for which the operator $-i d \widehat{\pi}(X)$ is positive. If $X$ is diagonal, this problem is solved by Theorem $\mathrm{\AA}$ if we put $\chi\left(\epsilon_{j}\right):=X_{j j}$, but the general case requires refined information on convex hulls of adjoint orbits (see Nee10 for similar problems). We plan to address this issue in a separate paper because it is of a functional analytic flavour, whereas the present paper is purely algebraic.

Notation. Throughout this paper, we denote by $\mathbb{N}=\{1,2, \ldots\}$ the set of positive natural numbers.

\section{Preliminaries}

2.1. Locally finite root systems. Let $J$ be an infinite set and let $V:=\mathbb{R}^{(J)} \subseteq \bar{V}:=\mathbb{R}^{J}$ be the free vector space over $J$, with canonical basis $\left\{e_{j} \mid j \in J\right\}$ and standard scalar product $\left(e_{j}, e_{k}\right)=\delta_{j k}$. In the dual space $V^{*} \cong \mathbb{R}^{J}$, we consider the linearly independent system $\left\{\epsilon_{j}:=e_{j}^{*} \mid j \in J\right\}$ defined by $\epsilon_{j}\left(e_{k}\right)=\delta_{j k}$.

Any infinite irreducible (possibly non-reduced) locally finite root system $\Delta$ can be described inside $V^{*}$ for some suitable set $J$, and is of one of the following types ([LN04, §8]):

$$
\begin{aligned}
A_{J} & :=\left\{\epsilon_{j}-\epsilon_{k} \mid j, k \in J, j \neq k\right\}, \\
B_{J} & :=\left\{ \pm \epsilon_{j}, \pm\left(\epsilon_{j} \pm \epsilon_{k}\right) \mid j, k \in J, j \neq k\right\}, \\
C_{J} & :=\left\{ \pm 2 \epsilon_{j}, \pm\left(\epsilon_{j} \pm \epsilon_{k}\right) \mid j, k \in J, j \neq k\right\}, \\
D_{J} & :=\left\{ \pm\left(\epsilon_{j} \pm \epsilon_{k}\right) \mid j, k \in J, j \neq k\right\}, \\
B C_{J} & :=\left\{ \pm \epsilon_{j}, \pm 2 \epsilon_{j}, \pm\left(\epsilon_{j} \pm \epsilon_{k}\right) \mid j, k \in J, j \neq k\right\} .
\end{aligned}
$$

For $X \in\{A, B, C, D, B C\}$, we will write $\Delta\left(X_{J}\right)$ for the above locally finite root system of type $X_{J}$. Note that the root systems of type $A_{J}, B_{J}, C_{J}$ and $D_{J}$ are reduced, whereas $\Delta\left(B C_{J}\right)$ is non-reduced.

2.2. The Weyl group of $\Delta$. Let $S_{J}$ denote the symmetric group on $J$, which we view as a subgroup of $\mathrm{GL}(\bar{V})$ with $w \in S_{J}$ acting as $w\left(e_{j}\right):=e_{w(j)}$. Given a permutation $w \in S_{J}$ with fixed-point set $I \subseteq J$, we call the set $J \backslash I$ the support of $w$. We denote by $S_{(J)} \leq S_{J}$ the subgroup of restricted permutations, namely, the set of $w \in S_{J}$ with finite support. Note that $S_{(J)} \leq \operatorname{GL}(\bar{V})$ stabilises $V$; we will also view $S_{(J)}$ as a subgroup of $\mathrm{GL}(V)$.

We next view $\{ \pm 1\}^{J} \subset \mathbb{R}^{J}$ as a subgroup of $\operatorname{GL}(\bar{V})$, acting by (componentwise) left multiplication: $\sigma\left(e_{j}\right)=\sigma_{j} e_{j}$ for $\sigma=\left(\sigma_{j}\right)_{j \in J} \in\{ \pm 1\}^{J}$. Given some $\sigma=\left(\sigma_{j}\right)_{j \in J} \in\{ \pm 1\}^{J}$, we call the subset $I=\left\{j \in J \mid \sigma_{j}=-1\right\}$ of $J$ the support of $\sigma$. We denote by $\{ \pm 1\}^{(J)}$ the set of all $\sigma \in\{ \pm 1\}^{J}$ with finite support. Again, we may also view $\{ \pm 1\}^{(J)}$ as a subgroup of $\operatorname{GL}(V)$. Finally, we let $\{ \pm 1\}_{2}^{(J)}$ denote the index 2 subgroup of $\{ \pm 1\}^{(J)}$, whose elements have a support of even cardinality.

Let $X \in\{A, B, C, D, B C\}$. We denote by $W=W\left(X_{J}\right)$ the Weyl group corresponding to $\Delta\left(X_{J}\right)$, which we view as a subgroup of $\operatorname{GL}(V)$ or $\operatorname{GL}(\bar{V})$. We then have the following descriptions (LN04, 
$\S 9]):$

$$
\begin{aligned}
& W\left(A_{J}\right)=S_{(J)}, \\
& W\left(B_{J}\right)=W\left(C_{J}\right)=W\left(B C_{J}\right)=S_{(J)} \ltimes\{ \pm 1\}^{(J)}, \\
& W\left(D_{J}\right)=S_{(J)} \ltimes\{ \pm 1\}_{2}^{(J)} .
\end{aligned}
$$

2.3. The positive energy condition. Let $X \in\{A, B, C, D, B C\}$ and set $W=W\left(X_{J}\right)$. Fix some tuples $\lambda=\left(\lambda_{j}\right)_{j \in J} \in \mathbb{R}^{J}$ and $\chi=\left(d_{j}\right)_{j \in J} \in \mathbb{R}^{J}$.

Definition 2.1. We say that the triple $(J, \lambda, \chi)$ satisfies the positive energy condition (PEC) for $W$ if the set $\lambda(W \cdot \chi-\chi)$ is bounded from below. Here, we view $\lambda$ as the linear functional

$$
\lambda: V \rightarrow \mathbb{R}: e_{j} \mapsto \lambda_{j},
$$

and $W \cdot \chi-\chi$ as a subset of $V$, by writing $\chi$ as $\chi=\sum_{j \in J} d_{j} e_{j} \in \bar{V}$. More precisely, recall from $\$ 2.2$ that any element of $W$ may be written as a product $\sigma w^{-1}$ for some $\sigma \in\{ \pm 1\}^{(J)}$ and some $w \in S_{(J)}$. Then

$$
\sigma w^{-1} \cdot \chi-\chi=\sum_{j \in J}\left(\sigma_{w^{-1}(j)} d_{j} e_{w^{-1}(j)}-d_{j} e_{j}\right)=\sum_{j \in J}\left(\sigma_{j} d_{w(j)}-d_{j}\right) e_{j} \in V .
$$

Note that for any $\sigma \in\{ \pm 1\}^{(J)}$ and $w \in S_{(J)}$, we have

$$
\lambda\left(\sigma w^{-1} \cdot \chi-\chi\right)=\sum_{j \in J} \lambda_{j}\left(\sigma_{j} d_{w(j)}-d_{j}\right) .
$$

In particular, given two disjoint finite subsets $\left\{i_{1}, i_{2}, \ldots, i_{k}\right\}$ and $\left\{j_{1}, j_{2}, \ldots, j_{k}\right\}$ of $J$, the product $w$ of the $k$ transpositions $\tau_{1}, \ldots, \tau_{k} \in S_{(J)}$, where $\tau_{s}$ interchanges $i_{s}$ and $j_{s}(s=1, \ldots, k)$, is an element of $S_{(J)}$ and we have

$$
\lambda(w \cdot \chi-\chi)=\lambda\left(w^{-1} \cdot \chi-\chi\right)=\sum_{s=1}^{k}\left(\lambda_{j_{s}}-\lambda_{i_{s}}\right)\left(d_{i_{s}}-d_{j_{s}}\right) .
$$

We record for future reference the following so-called rearrangement inequality.

Lemma 2.2. Let $a_{1} \leq a_{2} \leq \cdots \leq a_{n}$ and $b_{1} \leq b_{2} \leq \cdots \leq b_{n}$ be two non-decreasing sequences of real numbers. Let also $\left(c_{1}, \ldots, c_{n}\right)$ be a permutation of $\left(b_{1}, \ldots, b_{n}\right)$. Then

$$
\sum_{i=1}^{n} a_{i} b_{i} \geq \sum_{i=1}^{n} a_{i} c_{i} \geq \sum_{i=1}^{n} a_{i} b_{n+1-i}
$$

\section{A FEW DEFINITIONS AND NOTATIONS}

Fix some set $J$, as well as two tuples $\lambda=\left(\lambda_{j}\right)_{j \in J} \in \mathbb{R}^{J}$ and $\chi=\left(d_{j}\right)_{j \in J} \in \mathbb{R}^{J}$. We define the functions

We set

$$
D: J \rightarrow \mathbb{R}: j \mapsto d_{j} \quad \text { and } \quad \Lambda: J \rightarrow \mathbb{R}: j \mapsto \lambda_{j} .
$$

$$
m_{\min }=\inf \Lambda(J) \in \mathbb{R} \cup\{-\infty\} \quad \text { and } \quad m_{\max }=\sup \Lambda(J) \in \mathbb{R} \cup\{\infty\} .
$$

We call the triple $(J, \lambda, \chi)$ nontrivial if $\lambda$ is non-constant, so that $m_{\min } \neq m_{\max }$.

For each $n \in \mathbb{R}$, we set

$$
J_{n}:=\Lambda^{-1}(n),
$$

so that $J=\bigcup_{n} J_{n}$. Given some $r \in \mathbb{R}$, we also define the sets

$$
J_{n}^{>r}=\left\{j \in J_{n} \mid d_{j}>r\right\} \quad \text { and } \quad J_{n}^{<r}=\left\{j \in J_{n} \mid d_{j}<r\right\} .
$$

Finally, we let $\Lambda^{\infty}(J)$ denote the set of all $m \in \Lambda(J)$ such that $J_{m}$ is infinite, and we set

$$
\overline{\Lambda^{\infty}}(J):=\left(\Lambda^{\infty}(J) \cup\left\{m_{\min }, m_{\max }\right\}\right) \cap \mathbb{R} .
$$

Definition 3.1. We call $(J, \lambda, \chi)$ essentially bounded if for all $m \in \Lambda(J)$, the following two conditions are satisfied: 
(1) If $m \neq m_{\max }$, then $D\left(J_{m}\right)$ is bounded below.

(2) If $m \neq m_{\min }$, then $D\left(J_{m}\right)$ is bounded above.

Definition 3.2. Given a subset $I$ of $J$, we call $r \in \mathbb{R}$ an accumulation point for $I$ if either $r$ is an accumulation point for $D(I)$, or if $D\left(I^{\prime}\right)=\{r\}$ for some infinite subset $I^{\prime} \subseteq I$.

Definition 3.3. Assume that $(J, \lambda, \chi)$ is essentially bounded and nontrivial. Let $m \in \overline{\Lambda^{\infty}}(J)$.

(1) If $m \neq m_{\min }, m_{\max }$, then $D\left(J_{m}\right)$ is bounded, and hence $J_{m}$ possesses an accumulation point. In this case, we let $r_{m}^{\min }$ and $r_{m}^{\max }$ respectively denote the minimal and maximal accumulation points of $J_{m}$.

(2) If $m=m_{\text {min }}$, then $D\left(J_{m}\right)$ is bounded below. If $J_{m}$ has an accumulation point, we let $r_{m}^{\min }$ denote the minimal one. Otherwise, we set $r_{m}^{\min }=\infty$.

(3) If $m=m_{\max }$, then $D\left(J_{m}\right)$ is bounded above. If $J_{m}$ has an accumulation point, we let $r_{m}^{\max }$ denote the maximal one. Otherwise, we set $r_{m}^{\max }=-\infty$.

The following lemma, which easily follows from the definitions, provides the geometric picture to be kept in mind for the rest of this paper.

Lemma 3.4. Assume that $(J, \lambda, \chi)$ is essentially bounded and nontrivial. Let $m \in \overline{\Lambda^{\infty}}(J)$.

(1) If $m \neq m_{\min }, m_{\max }$, then for each $\epsilon>0$, there is some finite subset $I_{\epsilon} \subset J_{m}$ such that

$$
D\left(J_{m} \backslash I_{\epsilon}\right) \subseteq\left[r_{m}^{\min }-\epsilon, r_{m}^{\max }+\epsilon\right]
$$

(2) If $m=m_{\min }$ and $r_{m}^{\min } \neq \infty$, then for each $\epsilon>0$, there is some finite subset $I_{\epsilon} \subset J_{m}$ such that

$$
D\left(J_{m} \backslash I_{\epsilon}\right) \subseteq\left[r_{m}^{\min }-\epsilon, \infty[\text {. }\right.
$$

(3) If $m=m_{\max }$ and $r_{m}^{\max } \neq-\infty$, then for each $\epsilon>0$, there is some finite subset $I_{\epsilon} \subset J_{m}$ such that

$$
\left.\left.D\left(J_{m} \backslash I_{\epsilon}\right) \subseteq\right]-\infty, r_{m}^{\max }+\epsilon\right] .
$$

Before proceeding with the study of the PEC, we need to introduce one more concept.

Definition 3.5. Given $r \in \mathbb{R}$, we call a subset $I_{r}$ of $J$ of the form $J_{n}^{<r}$ or $J_{n}^{>r}$ summable (with respect to $(J, \lambda, \chi))$ if

$$
\Sigma\left(I_{r}\right):=\sum_{j \in I_{r}}\left|d_{j}-r\right|<\infty .
$$

\section{Consequences of the PEC for $W\left(A_{J}\right)$}

In this section, we fix some infinite set $J$ and some tuples $\lambda=\left(\lambda_{j}\right)_{j \in J} \in \mathbb{R}^{J}$ and $\chi=\left(d_{j}\right)_{j \in J} \in \mathbb{R}^{J}$, and we assume that $(J, \lambda, \chi)$ satisfies the PEC for $W=W\left(A_{J}\right)$.

Lemma 4.1. Let $m, n \in \mathbb{R}$ with $m<n$.

(1) If $J_{m}^{<r}$ and $J_{n}^{>r}$ are both nonempty for some $r \in \mathbb{R}$, then $D\left(J_{m}^{<r}\right)$ and $D\left(J_{n}^{>r}\right)$ are bounded.

(2) If $J_{m}$ and $J_{n}$ are both nonempty, then $D\left(J_{m}\right)$ (resp. $D\left(J_{n}\right)$ ) is bounded below (resp. above).

Proof. To prove (1), assume for a contradiction that $D\left(J_{m}^{<r}\right)$ is unbounded, and for each $k \in \mathbb{N}$, let $j_{k} \in J_{m}^{<r}$ such that $d_{j_{k}}<-k$. Pick any $j \in J_{n}^{>r}$. Consider for each $k \in \mathbb{N}$ the transposition $\tau_{k} \in W$ exchanging $j$ and $j_{k}$. It then follows from (2.2) that

$$
\lambda\left(\tau_{k} \cdot \chi-\chi\right)=\left(\lambda_{j}-\lambda_{j_{k}}\right)\left(d_{j_{k}}-d_{j}\right)=(n-m)\left(d_{j_{k}}-d_{j}\right)<-(n-m)\left(k+d_{j}\right)
$$

for all $k \in \mathbb{N}$, contradicting the PEC for $W$. The proof that $D\left(J_{n}^{>r}\right)$ is bounded is similar.

We now turn to the proof of (2). Assume that $J_{m}$ and $J_{n}$ are nonempty. We prove that $D\left(J_{m}\right)$ is bounded below, the proof for $D\left(J_{n}\right)$ being similar. Let $r \in \mathbb{R}$ be such that $J_{n}^{>r}$ is nonempty. If $J_{m}^{<r}$ is empty, then $\inf D\left(J_{m}\right) \geq r$. If $J_{m}^{<r}$ is nonempty, then $D\left(J_{m}^{<r}\right)$ is bounded by (1), and hence $D\left(J_{m}\right)$ is bounded below, as desired.

Lemma 4.2. Let $m, n \in \mathbb{R}$ with $m<n$, and let $r \in \mathbb{R}$. Assume that $J_{n}^{>r}$ is infinite. Then $J_{m}^{<t}$ is finite for all $t<r$. 
Proof. Assume for a contradiction that $J_{m}^{<t}$ is infinite for some $t<r$. Let $\left\{i_{1}, i_{2}, \ldots\right\}$ (resp. $\left.\left\{j_{1}, j_{2}, \ldots\right\}\right)$ be an infinite countable subset of $J_{m}^{<t}$ (resp. $J_{n}^{>r}$ ). For each $k \in \mathbb{N}$, let $w_{k} \in W$ be the product of the $k$ transpositions $\tau_{1}, \ldots, \tau_{k}$, where $\tau_{s}$ interchanges $i_{s}$ and $j_{s}(s \in \mathbb{N})$. It then follows from (2.2) that

$$
\lambda\left(w_{k} \cdot \chi-\chi\right)=\sum_{s=1}^{k}\left(\lambda_{j_{s}}-\lambda_{i_{s}}\right)\left(d_{i_{s}}-d_{j_{s}}\right)=(n-m) \sum_{s=1}^{k}\left(d_{i_{s}}-d_{j_{s}}\right)<-(n-m)(r-t) k
$$

for all $k \in \mathbb{N}$, contradicting the PEC for $W$.

Lemma 4.3. Let $m, n \in \mathbb{R}$ with $m<n$. Then there exists at most one $r \in \mathbb{R}$ such that $J_{m}^{<r}$ and $J_{n}^{>r}$ are both infinite.

Proof. Assume that $J_{m}^{<r}$ and $J_{n}^{>r}$ are both infinite for two different values of $r$, say $r_{1}<r_{2}$. Using Lemma 4.2 with $r=r_{2}$ and $t=r_{1}$ then yields the desired contradiction.

Lemma 4.4. If $(J, \lambda, \chi)$ satisfies the PEC for $W\left(A_{J}\right)$, it is essentially bounded.

Proof. Let $m \in \Lambda(J)$. If $m \notin \Lambda^{\infty}(J)$, then $D\left(J_{m}\right)$ is finite, hence bounded, and there is nothing to prove. Assume now that $m \in \Lambda^{\infty}(J)$. If $m \neq m_{\max }$, then there is some $n \in \Lambda(J)$ such that $m<n$. Since $J_{n} \neq \emptyset$, Lemma 4.1(2) implies that $D\left(J_{m}\right)$ is bounded below, as desired. Similarly, if $m \neq m_{\min }$, so that there exists some $n \in \Lambda(J)$ with $m>n$, Lemma 4.1(2) implies that $D\left(J_{m}\right)$ is bounded above, proving the claim.

Proposition 4.5. Assume that $(J, \lambda, \chi)$ satisfies the PEC for $W=W\left(A_{J}\right)$. Let $m, n \in \overline{\Lambda^{\infty}}(J)$ be such that $m<n$. Then one of the following assertions holds.

(1) $r_{n}^{\max }<r_{m}^{\min }$. In this case, there is some $r \in \mathbb{R}$ such that $J_{m}^{<r}$ and $J_{n}^{>r}$ are both finite.

(2) $r_{n}^{\max }=r_{m}^{\min }$. In this case, $J_{m}^{<r_{m}^{\min }}$ and $J_{n}^{>r_{n}^{\max }}$ are both summable.

Proof. Note first that $(J, \lambda, \chi)$ is essentially bounded by Lemma 4.4 and nontrivial by hypothesis, so that $r_{m}^{\min }$ and $r_{n}^{\max }$ are defined.

If $m=m_{\min }$ and $r_{m}^{\min }=\infty$, so that $D\left(J_{m}\right)$ is bounded below and $J_{m}$ has no accumulation point, then $J_{m}^{<r}$ is finite for any $r \in \mathbb{R}$. Since in addition $D\left(J_{n}\right)$ is bounded above, so that $J_{n}^{>r}$ is finite for some large enough $r$, the statement (1) is satisfied. Similarly, if $n=m_{\max }$ and $r_{n}^{\max }=-\infty$, the statement (1) is satisfied, and we may thus assume from now on that $r_{m}^{\min }, r_{n}^{\max } \in \mathbb{R}$.

We distinguish three cases.

Case 1: $r_{n}^{\max }<r_{m}^{\min }$.

It then follows from Lemma 3.4 that $J_{m}^{<r}$ and $J_{n}^{>r}$ are both finite for any $r \in \mathbb{R}$ with $r_{n}^{\max }<r<r_{m}^{\min }$. Hence (1) is satisfied in this case.

Case 2: $r_{n}^{\max }=r_{m}^{\min }$.

Set $r=r_{n}^{\max }=r_{m}^{\min }$. We now prove that $J_{m}^{<r}$ and $J_{n}^{>r}$ must be both summable, so that (2) is satisfied. By Lemma 3.4 the sets $J_{m}^{<r-1 / k}$ and $J_{n}^{>r+1 / k}$ are finite for each $k \in \mathbb{N}$, so that $J_{m}^{<r}=$ $\bigcup_{k \in \mathbb{N}} J_{m}^{<r-1 / k}$ and $J_{n}^{>r}=\bigcup_{k \in \mathbb{N}} J_{n}^{>r+1 / k}$ are at most countable.

If $J_{m}^{<r}$ and $J_{n}^{>r}$ are both finite, there is nothing to prove. Assume now that at least one of $J_{m}^{<r}$ and $J_{n}^{>r}$ is infinite, say $J_{m}^{<r}$ (the other case being similar). Write $J_{m}^{<r}=\left\{i_{1}, i_{2}, \ldots\right\}$. We distinguish two cases.

Assume first that $J_{n}^{>r}$ is infinite. Write $J_{n}^{>r}=\left\{j_{1}, j_{2}, \ldots\right\}$. For each $k \in \mathbb{N}$, let $w_{k} \in W$ be the product of the transpositions $\tau_{1}, \ldots, \tau_{k}$, where $\tau_{s}$ is the transposition exchanging $i_{s}$ and $j_{s}(s \in \mathbb{N})$. It then follows from (2.2) that

$$
\lambda\left(w_{k} \cdot \chi-\chi\right)=\sum_{s=1}^{k}\left(\lambda_{i_{s}}-\lambda_{j_{s}}\right)\left(d_{j_{s}}-d_{i_{s}}\right)=(m-n) \sum_{s=1}^{k}\left(d_{j_{s}}-d_{i_{s}}\right) .
$$


In particular,

$$
\inf _{k \in \mathbb{N}}\left\{\lambda\left(w_{k} \cdot \chi-\chi\right)\right\}=-(n-m) \sum_{s=1}^{\infty}\left(d_{j_{s}}-r+r-d_{i_{s}}\right)=-(n-m)\left(\Sigma\left(J_{n}^{>r}\right)+\Sigma\left(J_{m}^{<r}\right)\right) .
$$

Hence $J_{m}^{<r}$ and $J_{n}^{>r}$ must be both summable, as desired.

Assume next that $J_{n}^{>r}$ is finite. In particular $J_{n}^{>r}$ is summable, and it thus remains to show that $J_{m}^{<r}$ is also summable. Fix some $\epsilon>0$ and some sequence $\left(\epsilon_{s}\right)_{s \in \mathbb{N}}$ of positive real numbers such that $\sum_{s \in \mathbb{N}} \epsilon_{s}<\epsilon$. Since $r$ is an accumulation point for $J_{n}$, there is some infinite countable subset $\left\{j_{1}, j_{2}, \ldots\right\} \subseteq J_{n}$ such that $d_{j_{s}} \geq \max \left(d_{i_{s}}, r-\epsilon_{s}\right)$ for each $s \in \mathbb{N}$. For each $k \in \mathbb{N}$, let $w_{k} \in W$ be the product of the transpositions $\tau_{1}, \ldots, \tau_{k}$, where $\tau_{s}$ is the transposition exchanging $i_{s}$ and $j_{s}(s \in \mathbb{N})$. It then follows from (2.2) that

$$
\lambda\left(w_{k} \cdot \chi-\chi\right)=\sum_{s=1}^{k}\left(\lambda_{i_{s}}-\lambda_{j_{s}}\right)\left(d_{j_{s}}-d_{i_{s}}\right)=(m-n) \sum_{s=1}^{k}\left(d_{j_{s}}-d_{i_{s}}\right) .
$$

In particular,

$$
\inf _{k \in \mathbb{N}}\left\{\lambda\left(w_{k} \cdot \chi-\chi\right)\right\}=-(n-m) \sum_{s=1}^{\infty}\left(d_{j_{s}}-r+r-d_{i_{s}}\right) \leq-(n-m)\left(-\epsilon+\Sigma\left(J_{m}^{<r}\right)\right) .
$$

Hence $J_{m}^{<r}$ must be summable, as desired.

Case 3: $r_{n}^{\max }>r_{m}^{\min }$.

Let $r \in \mathbb{R}$ be such that $r_{m}^{\min }<r<r_{n}^{\max }$. Since $r_{m}^{\min }$ is an accumulation point for $J_{m}$, the set $J_{m}^{<r}$ is infinite. Similarly, since $r_{n}^{\max }$ is an accumulation point for $J_{n}$, the set $J_{n}^{>r}$ is infinite. Since there are infinitely many $r \in \mathbb{R}$ with $r_{m}^{\min }<r<r_{n}^{\max }$, Lemma 4.3 then yields a contradiction in this case. This concludes the proof of the proposition.

\section{Characterisation of the PEC for $\lambda$ Bounded And discrete}

In this section, we let $J$ denote some infinite set and $\lambda=\left(\lambda_{j}\right)_{j \in J}$ and $\chi=\left(d_{j}\right)_{j \in J}$ some tuples in $\mathbb{R}^{J}$. In order to characterise the PEC for the triple $(J, \lambda, \chi)$, we will need to make some finiteness assumption on $\lambda$.

Definition 5.1. We call $\lambda$ bounded (resp. discrete) if the subset $\Lambda(J)$ of $\mathbb{R}$ is bounded (resp. discrete).

Definition 5.2. Given a set $J$ and a tuple $\lambda=\left(\lambda_{j}\right)_{j \in J} \in \mathbb{R}^{J}$, we define the following cones in $\mathbb{R}^{J}$ :

$$
\begin{aligned}
& C_{\min }\left(\lambda, A_{J}\right)=\left\{\left(d_{j}\right)_{j \in J} \in \mathbb{R}^{J} \mid \forall i, j \in J: \lambda_{i}<\lambda_{j} \Longrightarrow d_{i} \geq d_{j}\right\}, \\
& C_{\min }\left(\lambda, B_{J}\right)=\left\{\left(d_{j}\right)_{j \in J} \in \mathbb{R}^{J} \mid \forall j \in J: \lambda_{j} d_{j} \leq 0 \text { and } \forall i, j \in J:\left|\lambda_{i}\right|<\left|\lambda_{j}\right| \Longrightarrow\left|d_{i}\right| \leq\left|d_{j}\right|\right\} .
\end{aligned}
$$

We also define the sub-vector space $\ell^{1}(J)=\left\{\left(d_{j}\right)_{j \in J} \in \mathbb{R}^{J}\left|\sum_{j \in J}\right| d_{j} \mid<\infty\right\}$ of $\mathbb{R}^{J}$.

Proposition 5.3. Let $X \in\{A, B\}$ and set $W=W\left(X_{J}\right)$. For a triple $(J, \lambda, \chi)$, the following are equivalent:

(1) $\inf \lambda(W \cdot \chi-\chi)=0$, i.e., $\lambda(w \cdot \chi-\chi) \geq 0$ for all $w \in W$.

(2) $\chi \in C_{\min }\left(\lambda, X_{J}\right)$.

Proof. We first deal with the case $X=A$. If $\chi \notin C_{\min }\left(\lambda, A_{J}\right)$, then there exist some $i, j \in J$ with $\lambda_{i}<\lambda_{j}$ and $d_{i}<d_{j}$. Denoting by $w \in W$ the transposition exchanging $i$ and $j$, we deduce from (2.2) that

so that $\inf \lambda(W \cdot \chi-\chi)<0$.

$$
\lambda(w \cdot \chi-\chi)=\left(\lambda_{j}-\lambda_{i}\right)\left(d_{i}-d_{j}\right)<0,
$$

Assume conversely that $\chi \in C_{\min }\left(\lambda, A_{J}\right)$ and let $w \in W=S_{(J)}$. Let $I$ be some finite subset of $J$ containing the support of $w$. By assumption, we may then write $I=\left\{i_{1}, \ldots, i_{k}\right\}$ so that

$$
\lambda_{i_{1}} \leq \cdots \leq \lambda_{i_{k}} \quad \text { and } \quad d_{i_{1}} \geq \cdots \geq d_{i_{k}} .
$$


Together with (2.1), the rearrangement inequality then implies that

$$
\lambda\left(w^{-1} \cdot \chi-\chi\right)=\sum_{s=1}^{k} \lambda_{i_{s}}\left(d_{w\left(i_{s}\right)}-d_{i_{s}}\right) \geq \sum_{s=1}^{k} \lambda_{i_{s}}\left(d_{i_{s}}-d_{i_{s}}\right)=0 .
$$

Hence $\inf \lambda(W \cdot \chi-\chi)=0$, as desired.

We next deal with the case $X=B$. If $\chi \notin C_{\min }\left(\lambda, B_{J}\right)$, then either there exists some $j \in J$ such that $\lambda_{j} d_{j}>0$, or else $\lambda_{k} d_{k} \leq 0$ for all $k \in J$ and there exist some $i, j \in J$ with $\left|\lambda_{i}\right|<\left|\lambda_{j}\right|$ and $\left|d_{i}\right|>\left|d_{j}\right|$. In the first case, denoting by $\sigma \in W$ the element of $\{ \pm 1\}^{(J)}$ with support $\{j\}$, we deduce from (2.1) that

$$
\lambda(\sigma \cdot \chi-\chi)=-2 \lambda_{j} d_{j}<0 .
$$

In the second case, denoting by $w \in S_{(J)}$ the transposition exchanging $i$ and $j$, and by $\sigma=\left(\sigma_{k}\right)_{k \in J}$ an element of $\{ \pm 1\}^{(J)}$ with support in $\{i, j\}$ satisfying $\sigma_{i} \lambda_{i} d_{j}=-\left|\lambda_{i} d_{j}\right|$ and $\sigma_{j} \lambda_{j} d_{i}=-\left|\lambda_{j} d_{i}\right|$, we deduce from (2.1) that

$$
\lambda\left(\sigma w^{-1} \cdot \chi-\chi\right)=\lambda_{i}\left(\sigma_{i} d_{j}-d_{i}\right)+\lambda_{j}\left(\sigma_{j} d_{i}-d_{j}\right)=\left(\left|\lambda_{i}\right|-\left|\lambda_{j}\right|\right)\left(\left|d_{i}\right|-\left|d_{j}\right|\right)<0 .
$$

In both cases, we deduce that $\inf \lambda(W \cdot \chi-\chi)<0$.

Assume conversely that $\chi \in C_{\min }\left(\lambda, B_{J}\right)$ and let $w \in S_{(J)}$ and $\sigma=\left(\sigma_{j}\right)_{j \in J} \in\{ \pm 1\}^{(J)}$. Let $I$ be some finite subset of $J$ containing the supports of $w$ and $\sigma$. By assumption, we may then write $I=\left\{i_{1}, \ldots, i_{k}\right\}$ so that

$$
\left|\lambda_{i_{1}}\right| \leq \cdots \leq\left|\lambda_{i_{k}}\right| \text { and }\left|d_{i_{1}}\right| \leq \cdots \leq\left|d_{i_{k}}\right| \text {. }
$$

Since moreover $\lambda_{i_{s}} d_{i_{s}}=-\left|\lambda_{i_{s}} d_{i_{s}}\right|$ for all $s=1, \ldots, k$, we deduce from (2.1) and the rearrangement inequality that

$$
\lambda\left(\sigma w^{-1} \cdot \chi-\chi\right)=\sum_{s=1}^{k} \lambda_{i_{s}}\left(\sigma_{i_{s}} d_{w\left(i_{s}\right)}-d_{i_{s}}\right) \geq \sum_{s=1}^{k}\left|\lambda_{i_{s}}\right|\left(-\left|d_{w\left(i_{s}\right)}\right|+\left|d_{i_{s}}\right|\right) \geq \sum_{s=1}^{k}\left|\lambda_{i_{s}}\right|\left(-\left|d_{i_{s}}\right|+\left|d_{i_{s}}\right|\right)=0 .
$$

Hence inf $\lambda(W \cdot \chi-\chi)=0$, as desired.

Lemma 5.4. Let $X \in\{A, B\}$. Assume that $\lambda$ is bounded and that $\chi \in \ell^{1}(J)$. Then $(J, \lambda, \chi)$ satisfies the PEC for $W\left(X_{J}\right)$.

Proof. Write $\lambda=\left(\lambda_{j}\right)_{j \in J}$ and $\chi=\left(d_{j}\right)_{j \in J}$, so that $C:=\sum_{j \in J}\left|d_{j}\right|<\infty$. Set $M:=\sup _{j \in J}\left|\lambda_{j}\right|<\infty$. Then for all $\sigma=\left(\sigma_{j}\right)_{j \in J} \in\{ \pm 1\}^{(J)}$ and $w \in S_{(J)}$, we have

$$
\lambda\left(\sigma w^{-1} \cdot \chi-\chi\right)=\sum_{j \in J} \lambda_{j}\left(\sigma_{j} d_{w(j)}-d_{j}\right) \geq-M \sum_{j \in J}\left(\left|d_{w(j)}\right|+\left|d_{j}\right|\right)=-2 M C .
$$

Since $\sigma$ and $w$ were arbitrary, this proves the claim.

Lemma 5.5. Let $X \in\{A, B\}$. Assume that $\lambda$ is bounded. Then for all $\chi^{\prime} \in \ell^{1}(J)$, the triple $(J, \lambda, \chi)$ satisfies the PEC for $W\left(X_{J}\right)$ if and only if the triple $\left(J, \lambda, \chi+\chi^{\prime}\right)$ satisfies the PEC for $W\left(X_{J}\right)$.

Proof. Set $W=W\left(X_{J}\right)$, and assume that $(J, \lambda, \chi)$ satisfies the PEC for $W$. Since

$$
\inf \lambda\left(W \cdot\left(\chi+\chi^{\prime}\right)-\left(\chi+\chi^{\prime}\right)\right) \geq \inf \lambda(W \cdot \chi-\chi)+\inf \lambda\left(W \cdot \chi^{\prime}-\chi^{\prime}\right),
$$

the triple $\left(J, \lambda, \chi+\chi^{\prime}\right)$ then satisfies the PEC for $W$ by Lemma 5.4. Replacing $\chi$ by $\chi+\chi^{\prime}$ and $\chi^{\prime}$ by $-\chi^{\prime}$, the converse follows.

Note that, up to now, we only refered to the locally finite root systems of type $A_{J}$ and $B_{J}$. We now prove that these are indeed the only cases to be considered.

Lemma 5.6. Assume that $(J, \lambda, \chi)$ satisfies the PEC for $W\left(B_{J}\right)$ or $W\left(D_{J}\right)$. Then

$$
\sum_{j \in J_{+}}\left|\lambda_{j} d_{j}\right|<\infty \text {, where } J_{+}:=\left\{j \in J \mid \lambda_{j} d_{j}>0\right\} \text {. }
$$


Proof. Note first that since $W\left(D_{J}\right)=S_{(J)} \ltimes\{ \pm 1\}_{2}^{(J)} \leq W\left(B_{J}\right)$, the PEC for $W\left(B_{J}\right)$ implies the PEC for $W\left(D_{J}\right)$. We may thus assume that $(J, \lambda, \chi)$ satisfies the PEC for $W\left(D_{J}\right)$. If $J_{+}$is finite, there is nothing to prove. Assume now that $J_{+}$is infinite. For each finite subset $I \subset J^{+}$of even cardinality, let $\sigma_{I} \in\{ \pm 1\}_{2}^{(J)}$ with support $I$. Then

$$
\inf \lambda\left(W\left(D_{J}\right) \cdot \chi-\chi\right) \leq \lambda\left(\sigma_{I} \cdot \chi-\chi\right)=-2 \sum_{i \in I} \lambda_{i} d_{i}=-2 \sum_{i \in I}\left|\lambda_{i} d_{i}\right| .
$$

Since $\sum_{j \in J^{+}}\left|\lambda_{j} d_{j}\right|$ is the supremum of all sums $\sum_{i \in I}\left|\lambda_{i} d_{i}\right|$ with $I$ a finite subset of $J^{+}$of even cardinality, the claim follows.

Lemma 5.7. Assume that $(J, \lambda, \chi)$ satisfies the PEC for $W\left(D_{J}\right)$. Let $m, n \in \Lambda(J)$ with $|m|<|n|$. Then $D\left(J_{m}\right)$ is bounded.

Proof. Assume for a contradiction that $D\left(J_{m}\right)$ is unbounded, and choose some infinite countable set $\left\{i_{0}, i_{1}, \ldots\right\} \subseteq J_{m}$ such that $\left|d_{i_{s}}\right| \geq s$ for all $s \in \mathbb{N}$. Let also $j \in J_{n}$. Fix some $s \in \mathbb{N}$, and let $w \in S_{(J)}$ be the transposition exchanging $j$ and $i_{s}$. Let also $\sigma=\left(\sigma_{j}\right)_{j \in J} \in\{ \pm 1\}_{2}^{(J)}$ with support in $\left\{j, i_{0}, i_{s}\right\}$ be such that $m \sigma_{i_{s}} d_{j}=-\left|m d_{j}\right|$ and $n \sigma_{j} d_{i_{s}}=-\left|n d_{i_{s}}\right|$. It then follows from (2.1) that

$$
\begin{aligned}
\lambda\left(\sigma w^{-1} \cdot \chi-\chi\right) & =m\left(\sigma_{i_{0}} d_{i_{0}}-d_{i_{0}}\right)+m\left(\sigma_{i_{s}} d_{j}-d_{i_{s}}\right)+n\left(\sigma_{j} d_{i_{s}}-d_{j}\right) \\
& \leq 2\left|m d_{i_{0}}\right|+|m| \cdot\left(-\left|d_{j}\right|+\left|d_{i_{s}}\right|\right)+|n| \cdot\left(-\left|d_{i_{s}}\right|+\left|d_{j}\right|\right) \\
& =2\left|m d_{i_{0}}\right|-(|n|-|m|)\left(\left|d_{i_{s}}\right|-\left|d_{j}\right|\right) \\
& \leq 2\left|m d_{i_{0}}\right|-(|n|-|m|)\left(s-\left|d_{j}\right|\right) .
\end{aligned}
$$

Hence

$$
\inf \lambda\left(W\left(D_{J}\right) \cdot \chi-\chi\right) \leq 2\left|m d_{i_{0}}\right|-(|n|-|m|)\left(s-\left|d_{j}\right|\right) .
$$

As $s \in \mathbb{N}$ was arbitrary, this contradicts the PEC for $W\left(D_{J}\right)$, as desired.

Lemma 5.8. Let $X \in\{B, C, D, B C\}$, and assume that $\lambda$ is bounded and discrete. Then $(J, \lambda, \chi)$ satisfies the PEC for $W\left(X_{J}\right)$ if and only if it satisfies the PEC for $W\left(B_{J}\right)$.

Proof. For $X=B, C, B C$, there is nothing to prove. Since $W\left(D_{J}\right) \leq W\left(B_{J}\right)$, it is also clear that the PEC for $W\left(B_{J}\right)$ implies the PEC for $W\left(D_{J}\right)$. Assume now that $(J, \lambda, \chi)$ satisfies the PEC for $W\left(D_{J}\right)=S_{(J)} \ltimes\{ \pm 1\}_{2}^{(J)}$, and let us prove that it also satisfies the PEC for $W\left(B_{J}\right)=S_{(J)} \ltimes\{ \pm 1\}^{(J)}$.

If $\Lambda(J)=\{m\}$ is a one-element set and $(J, \lambda, \chi)$ satisfies the PEC for $W\left(D_{J}\right)$, then

$$
\inf \lambda\left(W\left(D_{J}\right) \cdot \chi-\chi\right)=\inf _{|F| \text { even }}\left\{-2 m \sum_{j \in F} d_{j}\right\}>-\infty
$$

implies

$$
\inf \lambda\left(W\left(B_{J}\right) \cdot \chi-\chi\right)=\inf _{F}\left\{-2 m \sum_{j \in F} d_{j}\right\}>-\infty,
$$

where the above infima are taken over finite subsets $F$ of $J$. We therefore assume that $|\Lambda(J)| \geq 2$.

We distinguish two cases. Assume first that $J$ has no accumulation point. Since $\Lambda(J)$ is finite, there exists some $m \in \Lambda^{\infty}(J)$. Thus $D\left(J_{m}\right)$ is unbounded. Lemma 5.7 then implies that $|m| \geq|n|$ for all $n \in \Lambda(J)$. Similarly, if $n \in \Lambda^{\infty}(J)$, then $D\left(J_{n}\right)$ is unbounded and hence $|n|=|m|$. In other words, $\Lambda^{\infty}(J) \subseteq\{ \pm m\}$ and $|m|=\max \left\{\left|m_{\min }\right|,\left|m_{\max }\right|\right\}$. Thus either $\Lambda^{\infty}(J)=\{m\} \subseteq\left\{m_{\min }, m_{\max }\right\}$ with $|m|=\max _{j \in J}\left|\lambda_{j}\right|$, or else $m \neq 0$ and $\Lambda^{\infty}(J)=\{ \pm m\}=\left\{m_{\min }, m_{\max }\right\}$.

Since $(J, \lambda, \chi)$ is essentially bounded by Lemma 4.4 and $|\Lambda(J)| \geq 2$, the sets $D\left(J_{m_{\max }}\right)$ and $D\left(J_{m_{\min }}\right)$ are respectively bounded above and below. As $J$ has no accumulation point, this implies that the sets $J_{m_{\max }}^{>0}$ and $J_{m_{\min }}^{<0}$ are finite. Note also that the set

$$
S=\left\{j \in J \mid \lambda_{j} \neq m_{\min }, m_{\max }\right\}
$$


is finite because $\Lambda^{\infty}(J) \subseteq\left\{m_{\min }, m_{\max }\right\}$ and $\Lambda(J)$ is finite. In particular, the tuple $\chi^{\prime}=\left(d_{j}^{\prime}\right)_{j \in J}$ defined by

$$
d_{j}^{\prime}= \begin{cases}d_{j} & \text { if } j \in S \cup J_{m_{\max }}^{>0} \cup J_{m_{\min }}^{<0}, \\ 0 & \text { otherwise }\end{cases}
$$

belongs to $\ell^{1}(J)$. Thus by Lemma [5.5, we may replace without loss of generality $\chi$ by $\chi-\chi^{\prime}$. In other words, we may assume that

$$
D\left(J_{m_{\min }}\right) \subseteq[0, \infty), \quad D\left(J_{m_{\max }}\right) \subseteq(-\infty, 0], \quad \text { and } \quad D\left(J_{n}\right)=\{0\} \quad \text { for all } n \neq m_{\min }, m_{\max } .
$$

If $J_{m_{\min }}$ (resp. $J_{m_{\max }}$ ) is finite, we may, by a similar argument, assume that $D\left(J_{m_{\min }}\right)=\{0\}$ (resp. $\left.D\left(J_{m_{\max }}\right)=\{0\}\right)$. On the other hand, if $J_{m_{\min }}$ (resp. $\left.J_{m_{\max }}\right)$ is infinite, then $D\left(J_{m_{\min }}\right)$ (resp. $D\left(J_{m_{\max }}\right)$ ) is unbounded by hypothesis, and hence $m_{\min } \leq 0$ (resp. $m_{\max } \geq 0$ ) by Lemma [5.6

If $\Lambda^{\infty}(J)=\{m\}$ with $|m|=\max _{j \in J}\left|\lambda_{j}\right|$, we may thus assume that $m D\left(J_{m}\right) \subseteq(-\infty, 0]$ and that $D\left(J_{n}\right)=\{0\}$ for all $n \neq m$. If $\Lambda^{\infty}(J)=\{ \pm m\}=\left\{m_{\min }, m_{\max }\right\}$ is a 2-element set, we may similarly assume that $\pm m D\left(J_{ \pm m}\right) \subseteq(-\infty, 0]$ and that $D\left(J_{n}\right)=\{0\}$ for all $n \neq \pm m$. In both cases, $\chi \in C_{\min }\left(\lambda, B_{J}\right)$. Hence $(J, \lambda, \chi)$ satisfies the PEC for $W\left(B_{J}\right)$ by Proposition 5.3.

We next assume that $J$ has some accumulation point $r \in \mathbb{R}$. Set $M=\sup _{j \in J}\left|\lambda_{j}\right|$. Let $\epsilon>0$ and let $S$ be an infinite subset of $J$ such that $D(S) \subseteq[r-\epsilon, r+\epsilon]$. Let $\sigma=\left(\sigma_{j}\right)_{j \in J} \in\{ \pm 1\}^{(J)}$ and $w \in S_{(J)}$, and let $I$ be some finite subset of $J$ containing the supports of $\sigma$ and $w$. Pick any $i \in S \backslash I$, and let $\tau=\left(\tau_{j}\right)_{j \in J} \in\{ \pm 1\}^{(J)}$ with support contained in $\{i\}$ be such that $\tau \sigma \in\{ \pm 1\}_{2}^{(J)}$. It then follows from (2.1) that

$$
\begin{aligned}
\lambda\left(\sigma w^{-1} \cdot \chi-\chi\right) & =\sum_{j \in J} \lambda_{j}\left(\sigma_{j} d_{w(j)}-d_{j}\right)=\sum_{j \in J} \lambda_{j}\left(\tau_{j} \sigma_{j} d_{w(j)}-d_{j}\right)-\lambda_{i}\left(\tau_{i} d_{i}-d_{i}\right) \\
& \geq \lambda\left(\tau \sigma w^{-1} \cdot \chi-\chi\right)-2 M(|r|+\epsilon) .
\end{aligned}
$$

Hence

$$
\inf \lambda\left(W\left(B_{J}\right) \cdot \chi-\chi\right) \geq \inf \lambda\left(W\left(D_{J}\right) \cdot \chi-\chi\right)-2 M(|r|+\epsilon) .
$$

This concludes the proof of the lemma.

Remark 5.9. For each $X \in\{A, B, C, D, B C\}$, let $C_{\min }\left(\lambda, X_{J}\right)$ denote the set of $\chi \in \mathbb{R}^{J}$ such that $\inf \lambda\left(W\left(X_{J}\right) \cdot \chi-\chi\right)=0$. Note that, by Proposition [5.3, this is consistent with Definition 5.2.

For $X \in\{C, B C\}$, we have $W\left(X_{J}\right)=W\left(B_{J}\right)$, and hence $C_{\min }\left(\lambda, X_{J}\right)=C_{\min }\left(\lambda, B_{J}\right)$. In order to determine $C_{\min }\left(\lambda, D_{J}\right)$, we associate to each $\chi=\left(d_{j}\right)_{j \in J} \in \mathbb{R}^{J}$ the (possibly empty) set

$$
I_{\lambda, \chi}^{\min }:=\left\{i \in J|| \lambda_{i}\left|=\inf _{j \in J}\right| \lambda_{j} \mid \text { and }\left|d_{i}\right|=\inf _{j \in J}\left|d_{j}\right|\right\} .
$$

Note that $C_{\min }\left(\lambda, B_{J}\right) \subseteq C_{\min }\left(\lambda, D_{J}\right)$ because $W\left(D_{J}\right) \leq W\left(B_{J}\right)$. One can then check, as in the proof of Proposition 5.3 (or directly from $\underline{\mathrm{HN} 12}$, Corollary 3.2]), that $\chi \in C_{\min }\left(\lambda, D_{J}\right)$ if and only if either $\chi \in C_{\min }\left(\lambda, B_{J}\right)$, or else $\sigma_{i} \chi \in C_{\min }\left(\lambda, B_{J}\right)$ for some $i \in I_{\lambda, \chi}^{\min }$, where $\sigma_{i} \in\{ \pm 1\}^{(J)}$ has support $\{i\}$. As this fact will not be needed in our characterisation of the positive energy condition (see Lemma [5.8), we leave it as an exercise.

We first characterise the PEC for $W\left(A_{J}\right)$.

Theorem 5.10. Let $J$ be a set, and let $\lambda=\left(\lambda_{j}\right)_{j \in J}$ and $\chi=\left(d_{j}\right)_{j \in J}$ be elements of $\mathbb{R}^{J}$. Assume that $\lambda$ is discrete and bounded. Then the following are equivalent:

(1) $(J, \lambda, \chi)$ satisfies the PEC for $W\left(A_{J}\right)$.

(2) $\chi \in C_{\min }\left(\lambda, A_{J}\right)+\ell^{1}(J)$.

Proof. The implication (2) $\Longrightarrow(1)$ readily follows from Proposition 5.3 and Lemma 5.5. Assume now that $(J, \lambda, \chi)$ satisfies the PEC for $W\left(A_{J}\right)$, and let us prove that, up to substracting from $\chi$ some element of $\ell^{1}(J)$, one has $\chi \in C_{\min }\left(\lambda, A_{J}\right)$. Since $\chi \in C_{\min }\left(\lambda, A_{J}\right)$ if $\lambda$ is constant, we may assume that $(J, \lambda, \chi)$ is nontrivial, that is, $m_{\min } \neq m_{\max }$. Moreover, note that $(J, \lambda, \chi)$ is essentially bounded by Lemma 4.4 . 
By assumption, $\Lambda(J)$ is finite. Write $\overline{\Lambda^{\infty}}(J)=\Lambda^{\infty}(J) \cup\left\{m_{\min }, m_{\max }\right\}=\left\{n_{0}, n_{1}, \ldots, n_{k}\right\}$ so that

$$
m_{\min }=n_{0}<n_{1}<\cdots<n_{k}=m_{\max }
$$

for some $k \geq 1$. Proposition 4.5 then implies that

$$
r_{n_{0}}^{\min } \geq r_{n_{1}}^{\max } \geq r_{n_{1}}^{\min } \geq \cdots \geq r_{n_{k-1}}^{\max } \geq r_{n_{k-1}}^{\min } \geq r_{n_{k}}^{\max }
$$

For each $t \in\{1, \ldots, k\}$, we set

$$
a_{t}= \begin{cases}\frac{1}{2}\left(r_{n_{t-1}}^{\min }+r_{n_{t}}^{\max }\right) & \text { if } r_{n_{t-1}}^{\min }, r_{n_{t}}^{\max } \in \mathbb{R} \\ r_{n_{t}}^{\max }+1 & \text { if } t=1, r_{n_{0}}^{\min }=\infty \text { and } r_{n_{1}}^{\max } \in \mathbb{R} \\ r_{n_{t-1}}^{\min }-1 & \text { if } t=k, r_{n_{k}}^{\max }=-\infty \text { and } r_{n_{k}-1}^{\min } \in \mathbb{R} \\ 0 & \text { if } t=k=1, r_{n_{0}}^{\min }=\infty \text { and } r_{n_{1}}^{\max }=-\infty\end{cases}
$$

so that

$$
r_{n_{0}}^{\min } \geq a_{1} \geq r_{n_{1}}^{\max } \geq r_{n_{1}}^{\min } \geq a_{2} \geq \cdots \geq a_{k-1} \geq r_{n_{k-1}}^{\max } \geq r_{n_{k-1}}^{\min } \geq a_{k} \geq r_{n_{k}}^{\max } .
$$

We also set $a_{0}:=\infty$ and $a_{k+1}:=-\infty$. Fix some $t \in\{0,1, \ldots, k\}$. We claim that the tuple $\chi_{t}=\left(d_{j}^{\prime}\right)_{j \in J}$ defined by

$$
d_{j}^{\prime}=\left\{\begin{aligned}
d_{j}-a_{t+1} & \text { if } j \in J_{n_{t}} \text { and } d_{j}<a_{t+1} \\
d_{j}-a_{t} & \text { if } j \in J_{n_{t}} \text { and } d_{j}>a_{t} \\
0 & \text { otherwise }
\end{aligned}\right.
$$

is in $\ell^{1}(J)$.

Let $I_{+}$(resp. $I_{-}$) denote the set of $j \in J_{n_{t}}$ such that $d_{j}>a_{t}$ (resp. $d_{j}<a_{t+1}$ ). We have to show that

$$
\sum_{j \in I_{+}}\left|d_{j}-a_{t}\right|<\infty \text { and } \sum_{j \in I_{-}}\left|d_{j}-a_{t+1}\right|<\infty .
$$

We prove this for $I_{+}$, the proof for $I_{-}$being similar. Since if $t=0$, the set $I_{+}$is empty, we may assume that $t \in\{1, \ldots, k\}$. Moreover, since $a_{t} \geq r_{n_{t}}^{\max }$, the set $I_{+}$is finite as soon as $a_{t}>r_{n_{t}}^{\max }$. Note that this includes in particular the case where $t=k$ and $r_{n_{k}}^{\max }=-\infty$, in which case $D\left(J_{n_{t}}\right)$ is bounded above and $J_{n_{t}}$ has no accumulation point, as well as the case where $t=1, r_{n_{0}}^{\min }=\infty$ and $r_{n_{1}}^{\max } \in \mathbb{R}$, in which case $a_{t}=r_{n_{t}}^{\max }+1>r_{n_{t}}^{\max }$. Hence we may also assume that $a_{t}=r_{n_{t}}^{\max } \in \mathbb{R}$ and that $r_{n_{t-1}}^{\min } \in \mathbb{R}$. But then $r_{n_{t-1}}^{\min }=a_{t}=r_{n_{t}}^{\max }$, and hence the conclusion follows from Proposition 4.5.

Thus $\sum_{t=0}^{k} \chi_{t} \in \ell^{1}(J)$. Hence, up to replacing $\chi$ by $\chi-\sum_{t=0}^{k} \chi_{t}$, we may assume that

$$
D\left(J_{n_{t}}\right) \subseteq\left[a_{t+1}, a_{t}\right] \text { for all } t=0,1, \ldots, k .
$$

We next define the tuple $\chi^{\prime}=\left(d_{j}^{\prime}\right)_{j \in J}$ by

$$
d_{j}^{\prime}= \begin{cases}d_{j}-a_{t} & \text { if } \lambda_{j} \in \Lambda(J) \backslash \overline{\Lambda^{\infty}}(J) \text { and } n_{t-1}<\lambda_{j}<n_{t}, \\ 0 & \text { otherwise. }\end{cases}
$$

Since the set of $j \in J$ with $\lambda_{j} \in \Lambda(J) \backslash \overline{\Lambda^{\infty}}(J)$ is finite, $\chi^{\prime} \in \ell^{1}(J)$. Moreover, it follows from (5.1) that $\chi-\chi^{\prime} \in C_{\min }\left(\lambda, A_{J}\right)$. This concludes the proof of the theorem.

Before characterising the PEC for $W\left(B_{J}\right)$, we need one more lemma. For a tuple $\nu=\left(\nu_{j}\right)_{j \in J} \in \mathbb{R}^{J}$, we put

$$
|\nu|:=\left(\left|\nu_{j}\right|\right)_{j \in J} \in \mathbb{R}^{J}
$$

Lemma 5.11. Assume that $(J, \lambda, \chi)$ satisfies the PEC for $W\left(B_{J}\right)$. Then $(J,-|\lambda|,|\chi|)$ satisfies the PEC for $W\left(A_{J}\right)$.

Proof. Let $w \in S_{(J)}$, and let $I$ be some finite subset of $J$ containing the support of $w$. Let $\sigma=$ $\left(\sigma_{j}\right)_{j \in J} \in\{ \pm 1\}^{(J)}$ with support in $I$ be such that $\lambda_{j} \sigma_{j} d_{w(j)}=-\left|\lambda_{j} d_{w(j)}\right|$ for all $j \in I$. It then follows from (2.1) that

$$
-|\lambda|\left(w^{-1} \cdot|\chi|-|\chi|\right)=-\sum_{j \in I}\left|\lambda_{j}\right| \cdot\left(\left|d_{w(j)}\right|-\left|d_{j}\right|\right) \geq \sum_{j \in I} \lambda_{j}\left(\sigma_{j} d_{w(j)}-d_{j}\right)=\lambda\left(\sigma w^{-1} \cdot \chi-\chi\right) .
$$


Hence

as desired.

$$
\inf \left(-|\lambda|\left(W\left(A_{J}\right) \cdot|\chi|-|\chi|\right)\right) \geq \inf \lambda\left(W\left(B_{J}\right) \cdot \chi-\chi\right)
$$

Theorem 5.12. Let $J$ be a set, and let $\lambda=\left(\lambda_{j}\right)_{j \in J}$ and $\chi=\left(d_{j}\right)_{j \in J}$ be elements of $\mathbb{R}^{J}$. Assume that $\lambda$ is discrete and bounded. Then the following are equivalent:

(1) $(J, \lambda, \chi)$ satisfies the PEC for $W\left(B_{J}\right)$.

(2) $\chi \in C_{\min }\left(\lambda, B_{J}\right)+\ell^{1}(J)$.

Proof. The implication $(2) \Longrightarrow(1)$ readily follows from Proposition 5.3 and Lemma 5.5. Assume now that $(J, \lambda, \chi)$ satisfies the PEC for $W\left(B_{J}\right)$ and let us prove that, up to substracting from $\chi$ some element of $\ell^{1}(J)$, one has $\chi \in C_{\min }\left(\lambda, B_{J}\right)$.

Since $(J,-|\lambda|,|\chi|)$ satisfies the PEC for $W\left(A_{J}\right)$ by Lemma 5.11, we know from Theorem 5.10 that

$$
|\chi| \in C_{\min }\left(-|\lambda|, A_{J}\right)+\ell^{1}(J) \text {. }
$$

Let $\sigma=\left(\sigma_{j}\right)_{j \in J} \in\{ \pm 1\}^{J}$ be such that $\sigma_{j} d_{j} \geq 0$ for all $j \in J$. In other words, $|\chi|=\sigma \chi$. Hence $\chi \in \sigma C_{\min }\left(-|\lambda|, A_{J}\right)+\sigma \ell^{1}(J)$. Note that $\sigma \ell^{1}(J)=\ell^{1}(J)$. Up to substracting from $\chi$ some element of $\ell^{1}(J)$, we may thus assume without loss of generality that

$$
\chi \in \sigma C_{\min }\left(-|\lambda|, A_{J}\right) .
$$

Note that we may in addition assume that $(J, \lambda, \chi)$ satisfies the PEC for $W\left(B_{J}\right)$ by Lemma [5.5. We deduce from (5.2) that $|\chi| \in C_{\min }\left(-|\lambda|, A_{J}\right)$, so that

$$
\forall i, j \in J:\left|\lambda_{i}\right|<\left|\lambda_{j}\right| \Longrightarrow\left|d_{i}\right| \leq\left|d_{j}\right| .
$$

On the other hand, Lemma 5.6 implies that

$$
\sum_{j \in J_{+}}\left|d_{j}\right|<\infty, \quad \text { where } J_{+}:=\left\{j \in J \mid \lambda_{j} d_{j}>0\right\} .
$$

In particular, the tuple $\chi^{\prime}=\left(d_{j}^{\prime}\right)_{j \in J}$ defined by

$$
d_{j}^{\prime}= \begin{cases}2 d_{j} & \text { if } j \in J_{+} \\ 0 & \text { otherwise }\end{cases}
$$

belongs to $\ell^{1}(J)$. Since $\chi-\chi^{\prime}=\sigma^{\prime} \chi$, where $\sigma^{\prime}=\left(\sigma_{j}^{\prime}\right)_{j \in J} \in\{ \pm 1\}^{J}$ has support $J_{+}$, the tuple $\chi-\chi^{\prime}$ still satisfies the condition (5.3), with $d_{j}$ replaced by $d_{j}-d_{j}^{\prime}$ for all $j \in J$. Therefore, up to substracting $\chi^{\prime} \in \ell^{1}(J)$ from $\chi$, we may assume that $\lambda_{j} d_{j} \leq 0$ for all $j \in J$ and that (5.3) holds, so that $\chi \in C_{\min }\left(\lambda, B_{J}\right)$, as desired.

\section{Proof of Theorem $\mathrm{A}$}

By LN04, the root system $\Delta$ of the locally finite split simple Lie algebra $\mathfrak{g}$ over $\mathbb{K}=\mathbb{C}$ is isomorphic to one of the root systems $\Delta=\Delta\left(X_{J}\right)$ for $X \in\{A, B, C, D\}$ described in 2.1 where the Cartan subalgebra $\mathfrak{h}$ (resp. a one-dimensional extension of $\mathfrak{h}$ if $X=A$ ) is identified with $V_{\mathbb{C}}:=V \otimes_{\mathbb{R}} \mathbb{C}=$ $\operatorname{span}_{\mathbb{C}}\left\{e_{j} \mid j \in J\right\}$. The identification of $V_{\mathbb{C}}$ with $\mathfrak{h}^{*}$ (resp. a one-dimensional extension of $\mathfrak{h}^{*}$ ) induced by the assignment $e_{j} \mapsto \epsilon_{j}(j \in J)$ yields in turn an identification of the Weyl group $W \leq \mathrm{GL}\left(\mathfrak{h}^{*}\right)$ of $\mathfrak{g}$ with the Weyl group $W\left(X_{J}\right)$ defined in $\$ 2.2$,

Let $\lambda \in \mathfrak{h}^{*}$ be discrete and bounded. Then the restriction of $\lambda$ to $V$ is real valued, and $\lambda$ is determined by the tuple $\left(\lambda_{j}\right)_{j \in J} \in \mathbb{R}^{J}$ defined by $\lambda_{j}=\lambda\left(e_{j}\right), j \in J$. Identifying $\lambda$ with this tuple, $\lambda$ is then discrete and bounded in the sense of Definition 5.1.

Similarly, the character $\chi: \mathbb{Z}[\Delta] \rightarrow \mathbb{R}$ is the restriction of a $\mathbb{Z}$-linear map

$$
\tilde{\chi}: \operatorname{span}_{\mathbb{Z}}\left\{\epsilon_{j} \mid j \in J\right\} \rightarrow \mathbb{R}: \epsilon_{j} \mapsto d_{j},
$$

and is thus determined by the tuple $\left(d_{j}\right)_{j \in J} \in \mathbb{R}^{J}$. Note that $\mathbb{Z}[\Delta]=\operatorname{span}_{\mathbb{Z}}\left\{\epsilon_{j} \mid j \in J\right\}$ in all cases, except for $\Delta=\Delta\left(A_{J}\right)$, in which case $\mathbb{Z}[\Delta]$ is the corank 1 submodule $\left\{\sum_{j \in J} x_{j} \epsilon_{j} \mid \sum_{j \in J} x_{j}=0\right\}$ of $\operatorname{span}_{\mathbb{Z}}\left\{\epsilon_{j} \mid j \in J\right\}$. Hence, either $\tilde{\chi}=\chi$, or else $\Delta=\Delta\left(A_{J}\right)$ and $\tilde{\chi}$ is determined by $\chi$ up to a 
constant. As $W\left(A_{J}\right)=S_{(J)}$, modifying $\tilde{\chi}$ by a constant does not modify the value of the infimum of $\tilde{\chi}\left(W\left(A_{J}\right) \cdot \lambda-\lambda\right)$. We may thus safely replace $\chi$ by $\tilde{\chi}$, which we identify with the tuple $\left(d_{j}\right)_{j \in J} \in \mathbb{R}^{J}$.

Finally, with the above identifications, we have for all $\sigma \in\{ \pm 1\}^{(J)}$ and $w \in S_{(J)}$ that

$$
\begin{aligned}
\chi(\sigma w \cdot \lambda-\lambda) & =\chi\left(\sum_{j \in J} \lambda_{j}\left(\sigma_{w(j)} \epsilon_{w(j)}-\epsilon_{j}\right)\right)=\sum_{j \in J} \lambda_{j}\left(\sigma_{w(j)} d_{w(j)}-d_{j}\right)=\sum_{j \in J} d_{j}\left(\sigma_{j} \lambda_{w^{-1}(j)}-\lambda_{j}\right) \\
& =\lambda\left(\sum_{j \in J} d_{j}\left(\sigma_{j} e_{w^{-1}(j)}-e_{j}\right)\right)=\lambda\left(w^{-1} \sigma \cdot \sum_{j \in J} d_{j} e_{j}-\sum_{j \in J} d_{j} e_{j}\right) \\
& =\lambda\left((\sigma w)^{-1} \cdot \chi-\chi\right),
\end{aligned}
$$

and hence

$$
\inf \chi(W \cdot \lambda-\lambda)=\inf \lambda(W \cdot \chi-\chi) .
$$

In particular, the condition inf $\chi(W \cdot \lambda-\lambda)>-\infty$ in the statement of Theorem $\mathrm{\AA}$ is equivalent to requiring the triple $(J, \lambda, \chi)$ with $\lambda=\left(\lambda_{j}\right)_{j \in J}$ and $\chi=\left(d_{j}\right)_{j \in J}$ to satisfy the PEC for $W=W\left(X_{J}\right)$ in the sense of Definition 2.1]

For $X=A$, Theorem $\mathrm{A}$ thus sums up Proposition 5.3 and Theorem 5.10 Since $C_{\min }\left(\lambda, B_{J}\right) \subseteq$ $C_{\text {min }}\left(\lambda, D_{J}\right)$ (see Remark [5.9) and since the PEC for $W\left(B_{J}\right), W\left(C_{J}\right)$ and $W\left(D_{J}\right)$ are equivalent by Lemma [5.8, the conclusion of Theorem $\mathrm{A}$ for $X \in\{B, C, D\}$ follows from Proposition 5.3 and Theorem 5.12 .

\section{REFERENCES}

[BR87] Ola Bratteli and Derek W. Robinson, Operator algebras and quantum statistical mechanics. 1, second ed., Texts and Monographs in Physics, Springer-Verlag, New York, 1987, $C^{*}$ - and $W^{*}$-algebras, symmetry groups, decomposition of states.

[HN12] Georg Hofmann and Karl-Hermann Neeb, On convex hulls of orbits of Coxeter groups and Weyl groups, preprint (2012), to appear in Münster Journal of Mathematics, http://arxiv.org/abs/1204.2095.

[LN04] Ottmar Loos and Erhard Neher, Locally finite root systems, Mem. Amer. Math. Soc. 171 (2004), no. 811, $\mathrm{x}+214$.

[Nee98] Karl-Hermann Neeb, Holomorphic highest weight representations of infinite-dimensional complex classical groups, J. Reine Angew. Math. 497 (1998), 171-222.

[Nee04] _ Infinite-dimensional groups and their representations, Lie theory, Progr. Math., vol. 228, Birkhäuser Boston, Boston, MA, 2004, pp. 213-328.

[Nee10] _ Semibounded representations and invariant cones in infinite dimensional Lie algebras, Confluentes Math. 2 (2010), no. 1, 37-134.

[Nee12] _ Semibounded representations of Hermitian Lie groups, Travaux mathématiques. Vol. XXI, Trav. Math., vol. 21, Fac. Sci. Technol. Commun. Univ. Luxemb., Luxembourg, 2012, pp. 29-109.

[Nee14] _ Positive energy representations and continuity of projective representations for general topological groups, Glasg. Math. J. 56 (2014), no. 2, 295-316.

[Nee15] _ Projective semibounded representations of doubly extended Hilbert-Lie groups, in preparation (2015).

[NS01] Karl-Hermann Neeb and Nina Stumme, The classification of locally finite split simple Lie algebras, J. Reine Angew. Math. 533 (2001), 25-53.

[Sch61] John R. Schue, Cartan decompositions for $L^{*}$ algebras, Trans. Amer. Math. Soc. 98 (1961), 334-349.

[Stu99] Nina Stumme, The structure of locally finite split Lie algebras, J. Algebra 220 (1999), no. 2, 664-693.

Department Mathematik, FAU Erlangen-Nuernberg, Cauerstrasse 11, 91058 Erlangen, Germany

E-mail address: marquis@math.fau.de

Department Mathematik, FaU Erlangen-Nuernberg, Cauerstrasse 11, 91058 Erlangen, Germany

E-mail address: neeb@math.fau.de 